\title{
Improving Glycaemic Control in Patients with Type 2 Diabetes Mellitus through A Peer Support Instant Messaging Service Intervention (Diabpeers): Study Protocol for a Randomized Controlled Trial
}

\author{
Elisabeth Höld ( $\nabla$ elisabeth.hoeld@fhstp.ac.at) \\ Fachhochschule St Pölten GmbH: Fachhochschule St Polten https://orcid.org/0000-0001-6576-2338 \\ Johanna Grüblbauer \\ Fachhochschule St Pölten GmbH: Fachhochschule St Polten \\ Martin Wiesholzer \\ Universitätsklinikum St Pölten: Universitatsklinikum St Polten \\ Daniela Wewerka-Kreimel \\ Fachhochschule St Pölten GmbH: Fachhochschule St Polten \\ Stefan Stieger \\ Karl Landsteiner Privatuniversität für Gesundheitswissenschaften: Karl Landsteiner Privatuniversitat fur Gesundheitswissenschaften \\ Werner Kuschei \\ Universitätsklinikum St Pölten: Universitatsklinikum St Polten \\ Philip Kisser \\ Österreichische Gesundheitskasse \\ Elisabeth Gützer \\ Österreichische Gesundheitskasse \\ Ursula Hemetek \\ Fachhochschule St Pölten GmbH: Fachhochschule St Polten \\ Astrid Ebner-Zarl \\ Fachhochschule St Pölten GmbH: Fachhochschule St Polten \\ Jürgen Pripfl \\ Fachhochschule St Pölten GmbH: Fachhochschule St Polten; Christian Doppler Forschungsgesellschaft
}

\section{Research Article}

Keywords: Diabetes Mellitus Type 2, Instant Messaging Service, Peer Support, Randomized Controlled Trial, Austria, Diabetes SelfManagement (Support), Social Support, Glycemic Control, Quality of Life

Posted Date: December 10th, 2021

DOI: https://doi.org/10.21203/rs.3.rs-942223/v1

License: (c) (1) This work is licensed under a Creative Commons Attribution 4.0 International License. Read Full License 


\section{Abstract}

Background: the context and purpose of the study

Diabetes mellitus is one of the four priority non-communicable diseases worldwide. It can lead to serious long-term complications and produces significant costs. Due to the chronicle character of the disease, it requires continuous medical treatment and good therapy adherence of those suffering. Therefore, diabetes self-management education (DSME) (and support DSMES) plays a significant role to increase patient's self-management capacity and improve diabetes therapy. Research indicates that these outcomes might be difficult to maintain. Consequently, effective strategies to preserve the positive effects of DSMES are needed. Preliminary results show that peer support, which means support from a person who has experiential knowledge of a specific behaviour or stressor and similar characteristics as the target population, is associated with better outcomes in terms of $\mathrm{HbA}_{1 \mathrm{c}}$, cardiovascular disease risk factors or selfefficacy at lower cost compared to standard therapy. Peer-supported instant messaging services (IMS) approaches have significant potential for diabetes management because support can be provided easily and prompt, is inexpensive, and needs less effort to attend compared to standard therapy. The major objective of the study is to analyse the impact of a peer-supported IMS intervention in addition to a standard diabetes therapy on the glycaemic control of type 2 diabetic patients.

Methods: how the study will be performed

A total of 205 participants with type 2 diabetes mellitus will be included and randomly assigned to intervention or control group. Both groups will receive standard therapy, but the intervention group will participate in the peer-supported IMS intervention, additionally. The duration of the intervention will last for seven months, followed by a follow-up of seven months. Biochemical, behavioural and psychosocial parameters will be measured before, in the middle, and after the intervention as well as after the follow-up.

Discussion: a brief summary and potential implications

Diabetes mellitus type 2 and other non-communicable diseases put healthcare systems worldwide to the test. Peer-supported IMS interventions in addition to standard therapy might be part of new and cost-effective approaches to support patients independent from time and place.

Trial registration: If your article reports the results of a health care intervention on human participants, it must be registered in an appropriate registry and the registration number and date of registration should be in stated in this section. If it was not registered prospectively (before enrollment of the first participant), you should include the words 'retrospectively registered'. See our editorial policies for more information on trial registration.

ClinicalTrials.gov Identifier: NCT04797429

Date of registration: 15 March 2021

\section{Introduction}

\section{Background and rationale $\{6 a\}$}

SPIRIT guidance: Description of research question and justification for undertaking the trial, including summary of relevant studies (published and unpublished) examining benefits and harms for each intervention.

Diabetes mellitus is one of the four priority non-communicable diseases worldwide (1). Globally, 463 million adults suffered from diabetes mellitus (9.3\%) in 2019 and an increase of $51 \%$ of the prevalence is anticipated by 2045.59 million adults are affected at the European level (8.9\%) (2), while Austria shows a similar pattern: estimates range from 515,000 to 809,000 Austrians (7-11\% of all inhabitants) suffering from diabetes (3). In type 2 diabetes mellitus, which is the most common type of diabetes, hyperglycemia is the result of an inadequate production of insulin and insulin resistance, which means that the body cannot fully respond to insulin (2). Type 2 diabetes is most commonly observed in adults over the age of 40 (4).

Diabetes can lead to serious long-term complications such as cardiovascular diseases, blindness, or the amputation of lower extremities as well as lower quality of life, poorer mental health like greater levels of depression, and reduced life expectancy (2). Diabetes produces significant direct and indirect costs: 1.31 trillion USD in 2015 worldwide (5), 327 billion USD in 2017 in the USA (6), or 1.94 billion EUR even in Austria in 2014 (3). 
Due to the chronic nature of diabetes, the disease requires continuous therapy, regular medical check-ups and good therapy adherence. Patient self-management behaviors in particular play an important role in the therapy of the disease: while healthcare professionals are responsible for only $5 \%$ of diabetes therapy, $95 \%$ are in the hands of the patients themselves, including, e.g., eating habits, physical activity, medication adherence, stress control, and disease monitoring (7). Therefore, diabetes self-management education (DSME) (and support DSMES) play(s) a significant role in increasing a patient's self-management capacity and improving diabetes therapy (8-11). Self-management refers to the patient's ability to manage symptoms, therapy, physical and psychosocial consequences, and lifestyle changes associated with living with a disease (12). Consequently, DSME(S) is the process of facilitating skill, knowledge and abilities in diabetes self-care (11).

On the one hand, DSMES has been shown to improve clinical disease parameters like glycated hemoglobin $\left(\mathrm{HbA}_{1 \mathrm{c}}\right)(13-15)$, which reflects the average plasma glucose level over the previous 8-12 week and is the preferred test for diabetes control (16), and even mortality (17). On the other hand, research indicates that these outcomes might be difficult to maintain $(18,19)$ and seem to decline one to three months after the end of DSME(S) (20). Consequently, effective strategies to preserve the effects of DSME(S) are needed.

Social support, defined as "an exchange of resources between at least two persons aimed at increasing the wellbeing of the receiver" (21), is a well-known predictor of diabetes self-management, improves self-efficacy, adherence and glycemic control $(22,23)$, and reduces healthcare costs (24). Social support can be provided by several stakeholders like healthcare professionals, family members, friends or others living with the same disease (e.g., peers) (22).

Preliminary results show that peer support, which means support from a person who has experiential knowledge of a specific behavior or stressor and similar characteristics as the target population (25), is associated with better outcomes in terms of $\mathrm{HbA}_{1 \mathrm{c}}$, cardiovascular disease risk factors and self-efficacy (26-28) at lower cost (26) compared to standard therapy. Although those results are promising, research on peer support in diabetes care is still in its infancy $(29,30)$.

Peer support can be provided in various ways: face to face, telephone-based, web-based (29), or via other new technologies. New technologies in particular provide ideal and innovative means for peer support in diabetes management. Mobile Health (mHealth), which belongs to the group of information and communication technologies for health (World Health Organization, 2021), supports medical practice using mobile devices such as mobile phones, monitoring devices, personal digital assistants, and other wireless devices (31). Indeed, mobile phones have significant potential to serve as universal devices for diabetes management (32). However, methodologically sound evaluation studies assessing the impact of mHealth are scarce (Kitsiou et al., 2017; Wang et al., 2020) and data security as well as other ethical concerns are often not sufficiently considered $(32,35)$. Furthermore, most of the research on mHealth for diabetes focuses on support via apps or social media mainly provided by health professionals $(33,34,36,37)$. When it comes to support from peers or support provided via instant messenger services (IMS), less research is available. This is surprising because peer support IMS has particular advantages compared to other mHealth solutions:

- In 2020, the majority (72.9\%) of Austrians aged 45-64 who were mostly affected by the onset of type 2 diabetes used IMS, while only $27.5 \%$ used other social media tools (38).

- Off-the-shelf IMS technology is available which safeguards personal data protection, privacy and the associated ethical principles (e.g. grape: https://www.grape.io/de/).

- Compared to standard therapy, long-term support via IMS can be provided easily (26) and promptly (37), is inexpensive (26), and requires less effort to attend (39).

Generally, there is a need for high-quality randomized controlled trials (RCTs) on the impact of IMS telemedicine on health (40). Moreover, personal influences like genetic factors have not been properly considered yet: most existing studies focus mainly on average therapy effects, even though personalized medical approaches show that the response to therapy varies significantly between diabetic individuals $(41,42)$. In addition, theories from social sciences and psychology suggest that, depending on individual differences such as the regulatory mode, some individuals benefit more from mHealth interventions than others $(29,43,44)$. Furthermore, IMS users differ in terms of extraversion, neuroticism or conscientiousness (45), which might induce different health benefits related to personality traits.

The DiabPeerS study aims to implement a peer-supported IMS intervention for patients with type 2 diabetes mellitus and to analyze the effects of the intervention on diabetes mellitus type 2-related outcomes - especially $\mathrm{HbA}_{1 \mathrm{c}}$. Furthermore, DiabPeerS aims to gain further insight into the associations of personal influences on the individual use, acceptance and effects of peer-supported IMS interventions in diabetes therapy. 


\section{Objectives $\{7\}$}

SPIRIT guidance: Specific objectives or hypotheses.

The major objective of the study is to analyze the impact of a peer-supported IMS in addition to an antidiabetic therapy according to current Austrian guidelines (4) (hereafter referred to as 'standard therapy') on the glycemic control of type 2 diabetic patients. We hypothesize that peer-supported IMS improves maintenance of diabetes self-management and, hence, leads to longer-lasting improved disease-specific outcome parameters $\left(\mathrm{HbA}_{1 \mathrm{c}}\right)$ and higher quality of life. In particular, our main hypotheses are:

$\mathrm{H} 1$ : A peer-supported IMS intervention reduces $\mathrm{HbA}_{1 \mathrm{c}}$ of patients with type 2 diabetes mellitus compared to a standard therapy.

H2: A peer-supported IMS intervention helps to maintain diabetes self-management behaviors in patients with type 2 diabetes mellitus compared to a standard therapy.

H3: A peer-supported IMS intervention improves the quality of life of patients with type 2 diabetes mellitus compared to a standard therapy.

H4: A peer-supported IMS intervention improves medication adherence in patients with type 2 diabetes mellitus compared to standard therapy.

Furthermore, we want to assess the correlation between the specific personality trait of extraversion and the benefits of peer-supported IMS.

H5: Extraversion correlates positively with the benefits of peer-supported IMS intervention as measured by the frequency of IMS usage, quality of life, and $\mathrm{HbA}_{1 \mathrm{c}}$ levels.

\section{Trial design $\{8\}$}

SPIRIT guidance: Description of trial design including type of trial (eg, parallel group, crossover, factorial, single group), allocation ratio, and framework (eg, superiority, equivalence, noninferiority, exploratory).

The presented trial is a parallel-group, two-arm superiority RCT to evaluate the efficacy of a peer-supported IMS intervention for patients with diabetes mellitus type 2 in Lower Austria. Peer support via IMS in addition to standard therapy is compared with stand-alone standard therapy.

Moderators play a central role in peer-supported IMS intervention because they initiate and moderate the IMS exchange and discussion. They are patients with several years of disease and therapy experience because they need personal and practical experience in order provide supportive and credible coaching. All moderators receive prior training and guiding material (see $\{11 \mathrm{a}\}$ ). The moderators are supported by a dietitian at regular intervals and by the study team if needed. One moderator is responsible for one IMS group consisting of 14 participants.

The participants will be fully informed about all aspects of the trial and asked to sign a consent form. The participants will be randomized at a ratio of 1:1.

The duration of the intervention is seven months, followed by a seven-month follow-up period. The intervention will be performed twice to expand the recruitment phase. During the follow-up, the intervention group is expected to use the IMS tool without guidance by health professionals and both groups receive standard therapy.

\section{Methods: Participants, Interventions And Outcomes}

\section{Study setting $\{9\}$}

SPIRIT guidance: Description of study settings (eg, community clinic, academic hospital) and list of countries where data will be collected. Reference to where list of study sites can be obtained.

The trial will be implemented in Lower Austria, which is a federal state in northeastern Austria. It is the country's largest federal state and surrounds the Austrian capital Vienna. Lower Austria had 1,684,287 residents in 2020 (46), which reflects approximately 19\% of all inhabitants of Austria (47). 
Lower Austria has the second-highest prevalence of diabetes among all Austrian federal states. In this region, $5.7 \%$ of all residents above the age of 14 are affected by diabetes (3).

The trainings and medical examinations will be carried out at the St. Pölten University of Applied Sciences (located at the center of Lower Austria) and the hospitals in Wiener Neustadt (in the southeast of Lower Austria), Hollabrunn (in the northwest of Lower Austria) and Mauer/Amstetten (in the southwest of Lower Austria). This equal distribution throughout Lower Austria guarantees easy trial access for all participants. Biochemical analysis will be carried out at the University Hospital St. Pölten (located at the center of Lower Austria).

\section{Eligibility criteria $\{10\}$}

SPIRIT guidance: Inclusion and exclusion criteria for participants. If applicable, eligibility criteria for study centres and individuals who will perform the interventions (e.g., surgeons, psychotherapists).

Participants eligible for the trial must meet all of the following criteria during randomization:

Inclusion criteria participants (excluding moderators):

- older than 40 years

- living in Lower Austria

- diagnosed with type 2 diabetes mellitus according to the Austrian definition (4)

- $\mathrm{HbA}_{1 \mathrm{c}}$ of $\geq 6.5 \%$ (48 $\left.\mathrm{mmol} / \mathrm{mol}\right)$ in the most recent measurement

- undergoing standard therapy (4)

- have been receiving oral hyperglycemic agents for a maximum of one year prior to the start of the trial (first prescription of a minimum of two packages per year to exclude wrong prescriptions)

Inclusion criteria moderators:

The inclusion criteria for moderators differ from the inclusion criteria for other participants because moderators are required to be more experienced in the management of their disease in order to be able to authentically share personal and practical experiences and to effectively support participants.

Therefore, they should 1) be interested, 2) be engaged in therapy participation and judged by the practice team as being generally adherent to therapy, 3) have the capacity and commitment to undergo the training, 4) have a complete understanding of the importance of data security and patients' confidentiality, 5) cooperate with the dietitian and the study team if problems arise, 6 ) have the ability to fully understand the IMS strategy and the possibilities within the IMS tool, and 7) have several years of diseases experience (no newly diagnosed patients).

Therefore, their inclusion criteria are:

- older than 60 years

- living in the vicinity of the training location in St. Pölten, which means residing in St. Pölten, St. Pölten Land, Melk, Krems, or Lilienfeld

- diagnosed with type 2 diabetes mellitus according to the Austrian definition (4)

- $\mathrm{HbA}_{1 \mathrm{c}}$ of $\geq 6.5 \%(48 \mathrm{mmol} / \mathrm{mol})$ in the most recent measurement

- undergoing antidiabetic therapy according to the current guidelines (4)

- have been receiving oral hyperglycemic agents for a minimum of three years prior to the start of the trial (first prescription of a minimum of two packages per year to exclude wrong prescriptions)

- engaged participation (regular participation) in the Austrian disease management program 'Therapie aktiv - Diabetes im Griff', which is provided by the Austrian Health Insurance Fund

Exclusion criteria for participants as well as moderators:

- neurological or mental illness 
- hospitalization of more than 3 weeks during the intervention

- eye disorders that severely limit vision and, hence, inability to read the display (e.g., proliferative retinopathy or macular edema)

- severe illnesses such as kidney, liver, heart disease, or malignant cancer, which make a longer hospitalization likely

- illicit drug use or non-medical use of prescription drugs determined by a single-question screening test for drug use and drug use disorders in primary care (48)

- limited German language skills determined during the initial appointment concerning the informed consent

- pregnancy

\section{Who will take informed consent? $\{26 a\}$}

SPIRIT guidance: Who will obtain informed consent or assent from potential trial participants or authorised surrogates, and how (see Item 32).

Interested participants can register using an online registration form and a central study staff member - a dietitian or a nutritionist - will contact them afterwards.

During the initial appointment, which will be conducted online via MS Teams or face to face at the St. Pölten University of Applied Sciences, the staff member will explain the trial (background, protocol, different roles, importance of randomization and adherence) to potential participants and will review the consent form. Furthermore, the staff member will answer any questions arising. When the trial and its requirements are understood completely, the informed consent form will be signed by the participant. This can be done via electronic or handwritten signature.

All eligible participants must complete the consent procedure before enrolment and randomization. There are separate consent forms for general participants and for moderators.

\section{Compensation}

Participants and moderators will get a financial compensation in the amount of 50 EUR and a small gift after the last measurement. Furthermore, the results of the Bioelectrical Impedance Analysis measurements (BIA) in the context of body weight, body height, waist circumference, and blood pressure can be discussed with a dietitian for free after each measurement.

\section{Additional consent provisions for collection and use of participant data and biological specimens \{26b\}}

SPIRIT guidance: Additional consent provisions for collection and use of participant data and biological specimens in ancillary studies, if applicable.

No ancillary study will be conducted.

\section{Interventions}

\section{Explanation for the choice of comparators $\{6 \mathrm{~b}\}$}

SPIRIT guidance: Explanation for choice of comparators.

For the intervention group, we will use the instant messaging tool grape, in addition to standard therapy. grape is an instant messaging application that is installed on the cell phone and works similar to WhatsApp, i.e., people can use grape to send each other messages, photos, etc. Unlike WhatsApp, grape is distributed by an Austrian company and the users' data are hosted by servers located in Europe in compliance with European data protection regulations. In addition, the use for companies is fee-based.

\section{Intervention description \{11a\}}

SPIRIT guidance: Interventions for each group with sufficient detail to allow replication, including how and when they will be administered.

The intervention is developed and conducted by an interdisciplinary team. Dietitians and moderators play an active role in the operational process of the intervention. 
Dietitians are part of the team that develops the training curricula and is responsible for the training of the moderators and participants. They will supervise all moderators once a month via online meetings. During these meetings, the dietitians support moderators, e.g., if specific knowledge concerning diabetes care is needed or in case of technical problems.

Moderators act as facilitators and coordinators but they do not give medical or disease-related advice. In order to moderate communication and exchange in the IMS group effectively and authentically, personal and practical disease-related experiences of the moderator and the readiness to share these experiences with the group are essential. Moderators receive nine hours of training on the content to be shared via IMS and communication strategies to facilitate group exchange, peer support and behavioral change.

Participation in the clinical trial will last 15 months (1 month training/first measurement, 7 months intervention, 7 months follow-up). 196 participants will be randomly assigned to one of two groups: the intervention group or the control group. Participants who are randomly assigned to the intervention group will attend a two-hour technical training in connection with the first measurement. In the course of this training, they will learn how to use the grape app (https://www.grape.io/de/). After this training, they will join a grape group (Instant Messaging Service group) with a maximum of 14 people for 14 months (seven months guided and seven without guidance). For the first seven months of the intervention, an empowerment-based IMS communication strategy for diabetes self-management peer support has been developed.

Moderators are provided with a detailed handbook on web-based diabetes-related content and communication strategies to share this content with the group.

The IMS strategy combines two aspects that are essential for IMS diabetes self-management peer support: 1. a communication strategy that takes into account the special requirement of IMS communication, i.e., approaches to raise engagement and activities in the group, and 2. a diabetes self-management support strategy to make sure that all topics which are known to be of importance for diabetes selfmanagement (49) are covered and shared in a way that enables and empowers participants in their disease management. Thus, the IMS communication strategy contains, on the one hand, the IMS strategy which also involves a communication strategy that considers the special requirements of IMS and peer-communication on how to react and act in certain situations, for example to achieve engagement. On the other hand, it entails a didactic plan which outlines how, in what form and how frequently diabetic content shall be shared with the participants by moderators (e.g., web links, exchange of personal experiences/opinions). This IMS strategy will be operationalized in form of the training curriculum.

The moderator and the way she or he communicates with the group plays a vital role in guiding the group, setting the tone and creating an atmosphere of trust and providing support. By introducing the moderators to certain communication strategies that are also displayed in detail in the guidebook and can be reflected upon in the monthly meeting with the dietitian, a trusting atmosphere in the IMS group should be facilitated.

After the intervention, a seven-month follow-up will be conducted. During the follow-up, the intervention group may use the IMS tool in a self-organized manner, i.e., without guidance by the dietitian, and continue to receive their standard therapy.

Participants who are assigned to the control group will continue to receive their standard therapy but will not be assigned to a grape group.

The intervention will be conducted twice to increase the recruitment phase. The first intervention will start in January 2022. As soon as the first cohort enters the unaccompanied phase, a second moderated cohort will start - in both cases accompanied by an equally large control group. In total, we will be in the field for 23 months. With the start of the first intervention, we will have as many intervention and control teams with 14 people each as there are registrations. With the second intervention date, the remaining persons (196 in total) form further intervention and control teams.

\section{Criteria for discontinuing or modifying allocated interventions $\{11 \mathrm{~b}\}$}

SPIRIT guidance: Criteria for discontinuing or modifying allocated interventions for a given trial participant (eg, drug dose change in response to harms, participant request, or improving/worsening disease).

Participants can revoke their willingness to participate at any time, even without giving reasons, and withdraw from the clinical study without this causing them any disadvantages for their further medical care. 
The principal investigator may terminate a participation in the study prematurely without obtaining the participant's consent beforehand. The reasons for this may be:

(a) participants do not longer meet the inclusion requirements of the clinical trial

b) the treating doctor reasons that continued participation in the clinical trial is not in the participant's best interest

(c) the study coordinator makes the decision to terminate the entire clinical trial, or to terminate only one subject's participation prematurely

The communication protocols (see also $\{12\}$ ) based on activities within the IMS tool grape are reviewed weekly. The results of these reviews are used - in addition to the planned analysis of the KPIs - to monitor the intervention group and to intervene if necessary. Thus, they help the study coordinator and selected study team members to stay informed and, in case of extraordinary events (e.g., mobbing), to decide whether to continue, modify, or prematurely terminate the work with certain participants, moderators or communication concepts. If a participant does not adhere to the communication rules (netiquette) previously agreed upon in each IMS group - even after several discussions - he or she will be excluded.

\section{Strategies to improve adherence to interventions $\{11 \mathrm{c}\}$}

SPIRIT guidance: Strategies to improve adherence to intervention protocols, and any procedures for monitoring adherence (eg, drug tablet return, laboratory tests).

The intervention consists of a peer-supported IMS intervention in addition to standard therapy. Therefore, adherence of participants to the IMS intervention pertains mainly to the IMS strategy, the measurements and the initial training sessions.

The first step to improve adherence is the holistic explanation of the trial to interested participants during the initial appointment $(50,51)$. In this appointment, sufficient time is scheduled in order to enable comprehensive understanding concerning the trial among participants.

Those who have greater responsibility - the moderators - will be individuals participating in the disease management program 'Therapie aktiv - Diabetes im Griff' (https://www.therapie-aktiv.at), which is provided by the Austrian Health Insurance Fund. Hence, they are already proven to be generally adherent and engaged in their disease management. These moderators will positively influence their group members through the role model effect.

A dietitian experienced in counseling will support the moderators if problems arise and they will supervise all moderators once a month via online meetings. During the supervision, the dietitian will reflect on the past month and discuss the next steps of the IMS strategy with the moderators. If necessary, the communication expert can provide further assistance during these meetings.

Furthermore, we expect an additional positive social effect: participants will identify with their grape group and, in the next step, with the DiabPeerS trial. Hence, they will be interested in a successful intervention and participate actively. Also, frequent personal contact between the central study staff (EH, MH, AEZ) and the participants throughout the whole trial (initial appointment, four measurements, supervision meetings) will support adherence (50).

The IMS strategy (see $\{11 \mathrm{a}\}$ ), the core element of this trial, aims to improve diabetes self-management. Taking into consideration that regular medical appointments and monitoring are central in diabetes self-management, we assume that this increasing competence will support adherence to intervention as well.

Additionally, clinical visits will be designed as convenient for the participants as possible $(50,51)$. The participants will have easy access to the locations of training sessions and measurements: they can choose one out of four central locations in Lower Austria for their measurements. These locations are well known and easily accessible: the St. Pölten University of Applied Sciences and hospitals in Wr. Neustadt, Hollabrunn, and Mauer/Amstetten. These locations are equally distributed over Lower Austria from a geographical point of view and can be reached comfortably by car as well as public transport. The training sessions for the participants will take place after the first measurement at the same location. The training sessions for the moderators will be held at the St. Pölten University of Applied Sciences. Therefore, only individuals from St. Pölten or the surrounding areas are eligible to become moderators. If a participant misses one measurement, it is possible to make a new appointment within a certain timeframe. 
Furthermore, study staff will call participants to schedule visits and send them reminders via SMS and e-mail 48 hours before the appointment. During each measurement, participants and moderators will have the opportunity to discuss their results, such as body composition, with an experienced dietitian, which is not very common in Austria and will increase engagement (50).

Additionally, participants and moderators will receive a financial compensation of 50 EUR and a small gift after the last measurement.

\section{Relevant concomitant care permitted or prohibited during the trial $\{11 \mathrm{~d}\}$}

SPIRIT guidance: Relevant concomitant care and interventions that are permitted or prohibited during the trial.

All participants should proceed with their standard therapy (4), and the moderato rs should continue to participate in the disease management program 'Therapie aktiv - Diabetes im Griff' (https://www.therapie-aktiv.at) (52) during the trial.

It is also possible for participants to subscribe to the disease management program 'Therapie aktiv - Diabetes im Griff' during the trial. This program was launched in Austria in 2007 and aims to organize long-term and high-quality care for patients with diabetes mellitus type 2. It is voluntary and free of charge for physicians as well as for patients. It implements evidence-based clinical guidelines and supports patient empowerment, regular medical examinations, and lifestyle advice (52). At the end of 2020, 1,4976 patients with diabetes mellitus type 2 and 296 doctors in Lower Austria - or 93,099 patients with diabetes mellitus type 2 and 1,917 doctors at the national level - participated in this disease management program according to internal data of the Austrian Health Insurance Fund.

\section{Provisions for post-trial care $\{30\}$}

SPIRIT guidance: Provisions, if any, for ancillary and post-trial care, and for compensation to those who suffer harm from trial participation.

Trial participation entails only minimal risk compared to standard therapy alone, which will be continued after the trial. Therefore, no special provisions for post-trial care are included.

\section{Outcomes $\{12\}$}

SPIRIT guidance: Primary, secondary, and other outcomes, including the specific measurement variable (eg, systolic blood pressure), analysis metric (eg, change from baseline, final value, time to event), method of aggregation (eg, median, proportion), and time point for each outcome. Explanation of the clinical relevance of chosen efficacy and harm outcomes is strongly recommended.

The primary outcome is the level of $\mathrm{HbA}_{1 \mathrm{c}}[\%]$ measured using TOSOH G8 (Sysmex Austria GmbH).

Secondary outcomes are:

Psychosocial parameters:

- Social support will be measured using the 'Fragebogen zur Sozialen Unterstützung' (F-SozU) (53): the F-SozU operationalizes social support as perceived or anticipated support from the social environment. The short form consists of the following subscales: 'emotional support', 'practical support', 'social integration', 'stress from the social network'. The F-SozU involves of 14 items using a five-point Likert scale with the endpoints ' 1 ' (does not apply) and ' 5 ' (accurate).

- Self-efficacy will be measured using the 'General Self-Efficacy Scale' (GSE) (54): the GES consists of ten items designed on a fourpoint Likert scale with the endpoints ' 1 ' (not at all true) and ' 4 ' (exactly true) and assesses optimistic self-beliefs to cope with several challenges in life.

- Depression will be measured using the 'Patient Health Questionnaire-9' (PHQ-9) (55): the PHQ-9 asks for all nine criteria of depression as defined in the Diagnostic and Statistical Manual of Mental Disorders (DSM-IV) using a four-point Likert scale with the endpoints 'O' (not at all) and ' 3 ' (nearly every day).

- Diabetes distress will be measure using the 'Diabetes Distress Scale' (DDS) (56): the DDS includes for dimensions of distress ('emotional burden', 'regimen distress', 'interpersonal distress', 'physician distress'). The DDS consists of 17 items using a six-point Likert scale with the endpoints ' 1 ' (not a problem) and ' 5 ' (a very serious problem).

- Quality of life will be measured using the 'Short-Form-Health Survey' (SF-12) (57): the SF-12 includes eight dimensions ('physical functioning', 'role limitations due to physical problems', 'bodily pain', 'vitality', 'general health perceptions' , 'social functioning', 'role 
limitations due to emotional problems', 'mental health'). The summary scores 'physical component summary' and 'mental component summary' (0-100 scales) can be calculated from the specified scales.

- Diabetes knowledge will be measured using the 'Diabetes Knowledge Test' (DKT) (58) with forward and retranslation: the DKT consists of 20 statements about diabetes which have to be rated as 'true', 'false' or 'don't know'. Based on the answers, a difficulty index (percent of patients who scored correctly) is calculated.

\section{Behavioral parameters:}

- Medication adherence will be measured using the 'A14-scale' (59): the A14 consists of 14 items of non-adherent behaviors phrased in a non-threatening and non-judgemental way using a five-item Likert scale with the endpoints ' 4 ' (never) to ' 0 ' (very often).

- Dietary behavior and alcohol consumption will be measured using the food frequency questionnaire developed for the German Health Examination Survey for Adults 2008-2011 (DEGS-FFQ) (60,61): the semi-quantitative DEGS-FFQ assess 'dietary behavior' including 'alcohol consumption' during the past four weeks.

- Smoking will be measured using the questionnaire 'Aktivrauchen - Kurzversion (Erwachsene)' (62): the questionnaire assesses the current smoking behavior per week and that of the last six months. Participants indicate the number of cigarettes, cigarillos, cigars, or amount of tobacco (grams) used for pipes or hand-rolled cigarettes.

- Physical activity will be measured using the 'International Physical Activity Questionnaire Short Form' (IPAQ-SF) (63,64): the IPAQ-SF asks seven questions to assess 'vigorous-intensity' and 'moderate-intensity' physical activity as well as 'walking' and 'sitting'. Participants indicate the time in minutes or hours for each activity level. Based on this information, three levels of physical activity (low, moderate, high) are calculated and expressed in metabolic equivalent of task (MET) minutes per week.

- Diabetes self-management behaviors will be measured using the 'Summary of Diabetes Self-Care Activities German' (SDSCA-G) (65): the SDSCA-G focuses on the past seven days related to the diabetes self-care activities 'nutrition', 'physical activities', 'blood glucose testing', 'foot care', and 'smoking'. The SDSCA-G consists of 11 items and participants mark the number of days the mentioned behavior was performed on an eight-point Likert scale with the endpoints ' 0 ' ( 0 days) to ' 7 ' ( 7 days). Whilst the first ten items are calculated to a score and four sub scores (diet, exercise, blood-glucose testing, foot care), the eleventh item focuses on smoking habits.

- Clinic and communication visits (health professional visits in the past six months, hospital stays in the past six months).

\section{Biochemical parameters:}

- Fasting blood glucose [mg/dl] as measured using Cobas 8000 (Roche Austria $\mathrm{GmbH}$ )

- Total cholesterol [mg/dl] as measured using Cobas 8000 (Roche Austria $\mathrm{GmbH}$ )

- High-density lipoprotein (HDL) [mg/dl] as measured using Cobas 8000 (Roche Austria GmbH)

- Low-density lipoprotein (LDL) [mg/dl] and triglycerides [mg/dl] as measured using Cobas 8000 (Roche Austria $\mathrm{GmbH}$ )

- Blood pressure [mmHg] as measured using Boso Medicus uno OA

- Body weight $[\mathrm{kg}]$ and body height [cm] to calculate the body mass index (BMl: body weight in kilograms/height in meters squared) as measured using the Seca bella 840 scale and Seca 214 stadiometer

- Body fat [\%] as measured using Aengus BIACORPUS RX 4004M and BodyComp V 9.0 Professional (both MEDI CAL HealthCare $\mathrm{GmbH}$, Karlsruhe)

- Waist circumference [cm] to calculate the waist-to-height ratio as measured using an ergonomic, step less, and extendible measuring tape

Additionally, demographic data and personality traits will be surveyed in the baseline assessment (T0). Demographic data include age, gender, education, living arrangement (marital status), income, employment status, immigration background (66), and clinical information (age at diagnosis, on insulin, other medical therapies, list of prescribed medications). Personal traits will be assessed using the Big Five Inventory German (BFI-2) (67). The BFI-2 captures 'extraversion', 'agreeableness', 'conscientiousness', 'negative emotionality' and 'openness', each represented by three sub-facets. The BFI-2 consists of 60 items using a five-item Likert scale with the endpoints ' 1 ' (strongly disagree) to ' 5 ' (strongly agree).

Additionally, participation in the disease management program 'Therapie aktiv - Diabetes im Griff' as well as pregnancy will be evaluated at each measurement. 
As part of the data export solution of the IMS communication data, an export script is provided in which individual rooms - these correspond to the groups assigned for the experiment - are listed. Such communication protocols containing the communication data of the individual groups can be retrieved on a daily basis. These data include, for example, the persons who have sent a post, sent texts and files, timestamps or quotes. This information will be used to quantify the IMS communication activities.

The posts will be evaluated by content analysis to explain and interpret the results of $(\mathrm{H} 2)$ diabetes self-management behaviors, (H3) quality of life of participants, and (H4) medication adherence of participants. The codebook will be developed in collaboration with medical professionals for the coding of adherence and non-adherence behaviors. The quantified data will then be made available for the in-depth analysis of questionnaire data and medical scores.

According to past research (68-70), personality traits such as extraversion can influence our communication behavior: extraverted individuals speak more often, make more eye contact, smile and nod more frequently than introverted persons. (H5) Therefore, we assume that extraversion will correlate positively with the number of posts as well as the number of reactions and emojis to posts from others.

\section{Participant timeline $\{13\}$}

SPIRIT guidance: Time schedule of enrolment, interventions (including any run-ins and washouts), assessments, and visits for participants. A schematic diagram is highly recommended (see figure at http://www.spirit-statement.org/publications-downloads/).

The flow diagram showing the participant timeline through the DiabPeerS study is presented in Fig.1. The SPIRIT figure for this trial is given in Fig.2.

\section{Sample size $\{14\}$}

SPIRIT guidance: Estimated number of participants needed to achieve study objectives and how it was determined, including clinical and statistical assumptions supporting any sample size calculations.

An a priori calculation was performed using G-Power (71) for the primary outcome variable $\mathrm{HbA}_{1 \mathrm{c}}$, using an ANCOVA at $5 \%$ level of significance and $80 \%$ power. Based on previous research, we assumed a small to medium effect size for group differences in $\mathrm{HbA}_{1 \mathrm{c}}$ means (partial eta squared $=0.05$, which corresponds to an effect size $f=0.229)(72-74)$. Considering a $30 \%$ dropout ratio, we will include 98 randomly assigned participants in each group (196 total). The intervention group will be subdivided into seven IMS groups made up of 14 participants and one moderator each. Therefore, nine moderators (seven plus two for the compensation of possible dropouts) will be additionally recruited (205 persons in total, 196 participants plus nine moderators). Although the moderators will participate in the assessments, their data will not be included in the analyses.

\section{Recruitment $\{15\}$}

SPIRIT guidance: Strategies for achieving adequate participant enrolment to reach target sample size.

The Austrian Health Insurance Fund will perform a FoKo database (Folgekosten database) query based on defined inclusion and exclusion criteria to identify potential participants in Lower Austria.

The FoKo database is a social insurance data warehouse that is used by all health insurance providers in Austria. FoKo combines several IT (accounting) systems of Austrian health providers in one instrument and thus enables various analyses. FoKo collects data related to medical assistance, medication, and incapacity to work as well as in-patient stays or patient transport and dental treatment. FoKo has registered approximately 1.2 million people eligible for services of the Austrian Health Insurance Fund in Lower Austria.

Based on FoKo database queries of the years 2018 and 2019, approximately 6,000 potential participants will be identified. The data of the Mother-Child-Booklet, which is part of the Austrian pregnancy and childhood examinations, allows to identify pregnant women (with gestational diabetes) in order to exclude them from the study (75). The Austrian Health Insurance Fund will contact all identified patients with diabetes mellitus type 2 by personal letter while strictly considering data privacy. Additionally, the Austrian Health Insurance Fund will inform all relevant physicians and internists in Lower Austria about the trial and ask them to inform their patients about the possibility to participate in the trial. Furthermore, the diabetes-specific self-help organizations ADA (https://aktive-diabetiker.at/) and ÖDV (https://www.diabetes.or.at/home/) will inform their members about the trial. Additionally, leaflets will be disseminated at the Customer Service Centers of the Austrian Health Insurance Fund and via the consortium member institutions' online channels, e.g., their websites. 


\section{Sequence generation $\{16 a\}$}

SPIRIT guidance: Method of generating the allocation sequence (eg, computer-generated random numbers), and list of any factors for stratification. To reduce predictability of a random sequence, details of any planned restriction (eg, blocking) should be provided in a separate document that is unavailable to those who enrol participants or assign interventions.

Eligible participants who have signed the informed consent will be randomized into the peer-supported IMS intervention group or the control group in a 1:1 ratio. Random assignment will be conducted using virtual urn randomization during the initial appointment.

\section{Concealment mechanism $\{16 \mathrm{~b}\}$}

SPIRIT guidance: Mechanism of implementing the allocation sequence (eg, central telephone; sequentially numbered, opaque, sealed envelopes), describing any steps to conceal the sequence until interventions are assigned.

For the allocation of the participants, a simple urn-based randomization is carried out. For this purpose, an urn, in our case a digital card set, is filled with 196 cards. On 98 of them, it says 'intervention group' and on 98 'control group'. A unique ID is assigned to each card. The participants draw the cards themselves. This gives them the feeling that they have made their own decision and makes it easier for them to accept it, even if they do not end up in the desired group.

\section{Implementation $\{16 c\}$}

SPIRIT guidance: Who will generate the allocation sequence, who will enrol participants, and who will assign participants to interventions.

The drawing of the participants takes place during the initial appointment for the informed consent. Since the participants come continuously and from all over Lower Austria, interviews are conducted both in person and via video calls. In order to make the ballot box drawing online and offline comparable, a computer-based drawing will take place in both cases, i.e., similar to a memory game. The test persons turn over one (of 196) cards and thus find out in which group they participate and, at the same time, receive their ID for the study. The drawing takes place without putting the cards back.

The maximum group size is 14 participants. When an intervention or control group is full, the recruitment of the next group will be started. The ID numbers are therefore not consecutive within the groups, but random. There is no upper limit regarding the number of participants for the first recruitment date.

\section{Assignment of interventions: Blinding}

\section{Who will be blinded $\{17 \mathrm{a}\}$}

SPIRIT guidance: Who will be blinded after assignment to interventions (eg, trial participants, care providers, outcome assessors, data analysts), and how.

Blinding of trial participants will not be possible because of the obvious differences between the intervention group and the control group. Blinding will be implemented for the medical staff and the outcome assessors of the measurements and the following analyses (see $\{19\})$.

\section{Procedure for unblinding if needed $\{17 \mathrm{~b}\}$}

SPIRIT guidance: If blinded, circumstances under which unblinding is permissible, and procedure for revealing a participant's allocated intervention during the trial.

It is an open-label study and therefore unblinding will not occur.

\section{Data collection and management}

\section{Plans for assessment and collection of outcomes $\{18 \mathrm{a}\}$}

SPIRIT guidance: Plans for assessment and collection of outcome, baseline, and other trial data, including any related processes to promote data quality (eg, duplicate measurements, training of assessors) and a description of study instruments (eg, questionnaires, 
laboratory tests) along with their reliability and validity, if known. Reference to where data collection forms can be found, if not in the protocol.

During the online registration, contact data of all interested individuals will be collected. The data will be deleted if the person disagrees to participate in the trial.

Data will be collected four times over a period of 14 months: at baseline (T0), three months after the start of the intervention (T1; T0+3 months), at the end of the intervention (T2; T0+7 months), and after the follow-up (T3; T0+14 months), including the collection of biochemical, psychosocial, and behavioral parameters as described in the subsection 'Outcomes (see $\{12\}$ )' during all four data collection points.

All parameters will be taken in a group testing setting with appropriate Coronavirus SARS-CoV-2 (COVID-19) safety measures, e.g., testing, vaccination, hygiene procedures by the staff of the St. Pölten University of Applied Sciences either at the university itself or at the Landesklinikum Wr. Neustadt, Hollabrunn, or Mauer/Amstetten.

The measurements will take place in the morning in a fasting state and with anthropometric measurements with light clothing. Anthropometric measurements will be conducted by dietitians. Blood pressure und blood samples will be taken by nurses. Fresh blood samples will be taken by the staff of the St. Pölten University of Applied Sciences and analyzed within a timeframe of three hours maximum by the University Hospital St. Pölten. Therefore, blood samples will be taken at the end of each data collection session and will be brought immediately to the University Hospital St. Pölten without interrupting the cold chain. The laboratory of the University Hospital St. Pölten is ISO 9001-certified and will handle all samples according to its quality management.

\section{Plans to promote participant retention and complete follow-up $\{18 \mathrm{~b}\}$}

SPIRIT guidance: Plans to promote participant retention and complete follow-up, including list of any outcome data to be collected for participants who discontinue or deviate from intervention protocols.

To minimize loss during the follow-up, and in order to promote participation in the follow-up measurements, the same strategy is implemented as for compliance with the intervention measurements (see $\{11 \mathrm{c}\}$ ): as convenient as possible, reminders $48 \mathrm{~h}$ ahead of the measurement, possibility to discuss the measurements' results with the dietitian, and a financial compensation paid after the last measurement.

During the follow-up, participants of the intervention group can use the messenger service and continue their communication with the group members and the moderator, hence, without support of the study team. The moderator will be instructed to motivate the group members to participate in the follow-up measurement. The relationship of trust and social support in the groups as well as with the study staff will support a higher degree of complete follow-up.

\section{Data management $\{19\}$}

SPIRIT guidance: Plans for data entry, coding, security, and storage, including any related processes to promote data quality (eg, double data entry; range checks for data values). Reference to where details of data management procedures can be found, if not in the protocol.

The participants agree that for the purpose and in the course of this study, the St. Pölten University of Applied Sciences will collect and process the following personal data: name, age, gender, education, income, occupational situation, housing situation, migration background, personality characteristics, clinical information such as age at diabetes diagnosis, social support, self-efficacy, depression, quality of life, diabetes knowledge, treatment adherence, diet and exercise behavior, nicotine and alcohol consumption, diabetes selfmanagement, medical consultations during the last six months, $\mathrm{HbA1c}$, fasting glucose, total cholesterol, $\mathrm{HDL}$, LDL, triglycerides, blood pressure, body weight and height, abdominal circumference, and body composition.

Furthermore, the participants agree that the University Hospital St. Pölten may transmit the following data in indirectly personalized (pseudonymized or encrypted) form to the St. Pölten University of Applied Sciences for the purpose of analyzing whether the intervention is effective with regard to the defined parameters compared to conventional therapy: HbA1c, fasting glucose, total cholesterol, HDL, LDL, triglycerides.

The participants' data will be pseudonymized and assigned a fixed identification code. This code will be stored safely in a passwordprotected file, accessible only by the study coordinator $(E H)$ and central staff members (UH, AEZ). The key for the coding is kept

Page 13/26 
separately and inaccessibly by the study coordinator. Thus, it is only possible for persons who are entrusted with the evaluation of the data to infer the identity of the participants.

Data acquisition and data processing are performed using commercial software. All data obtained are stored in computer files in encrypted form. The coding is done by means of consecutive numbering, from which only the allocation to the corresponding group, but in no case a conclusion as to the identity of the participant is possible.

Data will be stored and analyzed via the statistical software package SPSS at servers of the St. Pölten University of Applied Sciences, only used for this special study and not be passed on to third parties. The handling of the data complies with the European General Data Protection Regulation, the Austrian Data Protection Act and the recommendations of the Ethics Committee of Lower Austria.

To be able to merge the data from the paper-and-pencil questionnaires, anthropometric measurements and the blood pressure data, a defined coding system will be used. Therefore, the study coordinator will take part in every measurement and organize the participants and coding system. Hence, every participant will get his or her identification code and the questionnaires will be marked with this code at the beginning of each measurement. The identification code ensures that the study staff do not know to which group each participant belongs. Paper-and-pencil questionnaires will be checked by the study staff in terms of completeness and correctness related to the specifications of the questionnaires. These data will be transferred into SPSS manually and controlled by another member of the study team. Results of the biochemical analysis will be mailed via encrypted Excel files from the University Hospital St. Pölten to the St. Pölten University of Applied Sciences and imported into SPSS by the study coordinator. Blood samples will be disposed of and Excel files will be deleted after integration into SPSS. Furthermore, the St. Pölten University of Applied Sciences performs data back-ups on a daily basis. All signed informed consent forms as well as all collected paper-and-pencil sheets will be stored in a locked place, accessible only to the study coordinator and a central staff member (UH). All data will be stored at the St. Pölten University of Applied Sciences for ten years after the study's end. After that, all data will be destroyed.

To ensure data quality, the study coordinator will control the data regularly including checking the range of values or double data entry.

Data protection concerning the instant messaging tool will be met by using the instant messaging communication tool grape (https://www.grape.io/de/). grape has been chosen for its clear terms and conditions and data handling strategy. grape offers a fully transparent and documented communication infrastructure that stores cloud data in Austria.

grape is an Austrian technology start-up based in Vienna that provides a holistic messaging solution that allows companies to communicate securely and efficiently via chats, video, voice calls and screen sharing internally and externally. For our study, the grape communication platform (software solution) is hosted by a European data center near Vienna (ISO27001:2013 certified). The data export based on Docker is conducted in grape's data center and made available only to selected members of the study team (EH, AEZ, JG). The data are cleaned, and unstructured communication data will be structured using content analysis and MAXQDA (see \{12\}). Subsequently, these data will be pseudonymized and transferred into SPSS for further analysis.

Results of all analyses will be reported in an aggregated and strictly anonymous form.

\section{Confidentiality $\{27\}$}

SPIRIT guidance: How personal information about potential and enrolled participants will be collected, shared, and maintained in order to protect confidentiality before, during, and after the trial.

All collected data will be kept confidential as described in 'Data management' (see $\{19\})$. Only the study coordinator and central staff members (UH, AEZ) can open the password-protected file to link personal information and code. The final pseudonymized dataset in SPSS will be used by the study staff for analyses, publications, and reports only for this study.

\section{Plans for collection, laboratory evaluation and storage of biological specimens for genetic or molecular analysis in this trial/future use} $\{33\}$

SPIRIT guidance: Plans for collection, laboratory evaluation, and storage of biological specimens for genetic or molecular analysis in the current trial and for future use in ancillary studies, if applicable.

Not applicable as no biological specimens for genetic or molecular analysis will be collected.

\section{Statistical methods}

Page $14 / 26$ 
SPIRIT guidance: Statistical methods for analysing primary and secondary outcomes. Reference to where other details of the statistical analysis plan can be found, if not in the protocol.

Data will be analyzed using IBM SPSS Statistics 26 or greater (IMB Corporation, Armonk, NY, USA). The significance level will be set at $5 \%$. Generally, metric data will be checked for normal distribution using a histogram of each variable, as well as for skewness and kurtosis. To illustrate descriptive parameters, the arithmetic mean and the $95 \%$ confidence interval will be stated for metric data. Interval variables or nominal scaled variables will be shown as frequencies. Repeated measures ANCOVA will be used to compare the control and intervention groups concerning primary and secondary outcome parameters. Non-normal metric data will be analyzed using the Mann-Whitney U test, the Kruskal-Wallis signed rank test or the Friedman-Test (76).

\section{Interim analyses $\{21 b\}$}

SPIRIT guidance: Description of any interim analyses and stopping guidelines, including who will have access to these interim results and make the final decision to terminate the trial.

There will be no planned interim analysis or stopping guidelines for medical reasons besides the exclusion criteria (see $\{10\}$ ) because no potentially harmful outcomes are expected based on the peer-supported IMS intervention. Nevertheless, participants can be excluded from the intervention group if they do not follow the rules of interaction set and agreed upon in the IMS group (see \{11b\}).

\section{Methods for additional analyses (e.g. subgroup analyses) \{20b\}}

SPIRIT guidance: Methods for any additional analyses (eg, subgroup and adjusted analyses).

As we want to assess the association between specific personality traits and the benefits of peer-supported IMS, we use validated and published questionnaires, which we repeatedly distribute to all experimental and control groups at different times of the study.

In addition to the observed communication behavior, we use the information from the questionnaires (see $\{12\})$ to test the hypotheses and in particular related to $(\mathrm{H} 2)$ diabetes self-management behaviors, $(\mathrm{H} 3)$ quality of life of participants, and (H4) medication adherence as well as (H5) the level of extraversion.

\section{Methods in analysis to handle protocol non-adherence and any statistical methods to handle missing data $\{20 \mathrm{c}\}$}

SPIRIT guidance: Definition of analysis population relating to protocol non-adherence (eg, as randomised analysis), and any statistical methods to handle missing data (eg, multiple imputation).

All participants' data will be analyzed following the intention-to-treat principle. Scores for scales with at least $80 \%$ of items responded will be analyzed. For these cases, the nearest neighbour method will be used to replace missing data points. Furthermore, we will check the randomness of missing data by calculating the MCAR (missing completely at random) classification.

\section{Plans to give access to the full protocol, participant level-data and statistical code $\{31 \mathrm{c}\}$}

SPIRIT guidance: Plans, if any, for granting public access to the full protocol, participant-level dataset, and statistical code.

It is not planned to give third parties access, neither to the full protocol, nor the participant data, nor the statistical code.

\section{Oversight and monitoring}

\section{Composition of the coordinating centre and trial steering committee $\{5 \mathrm{~d}\}$}

Trials guidance: Provide information on the composition, roles and responsibilities of the coordinating centre and trial steering committee and all groups providing day to day support for the trial. There will always be a group running the trial day-to-day and providing organisational support and knowing how often they will meet, plus information on other committees providing oversight such as a Trial Steering Committee, and how often they will meet over the course of the trial, is what we need for item $5 \mathrm{~d}$. We do not need names of staff.

SPIRIT guidance: Composition, roles, and responsibilities of the coordinating centre, steering committee, endpoint adjudication 
committee, data management team, and other individuals or groups overseeing the trial, if applicable (see Item 21a for data monitoring committee).

The complexity of diabetes therapy demands interdisciplinary teams and innovative treatment approaches. This consortium meets these requirements and combines life sciences, nutritional sciences \& dietetics, human medicine, psychology and with media economics, communications and sociology in a unique constellation. Responsibilities are assigned on the basis of the expertise of the respective study team members. Responsible persons for the following areas are defined as relevant to the study: Instant Messenger Interventions, Dietetics, Medical Measurements, Recruitment, and Biochemical Analyses and Statistics. In addition, two persons are available as supervisors for the areas of Nutrition and Communication, respectively, and a medical doctor is also part of the study team.

Meetings of the entire study team are held every two months or more frequently when meetings are needed, or in subgroups. In addition, experts are available on request. Decisions are made democratically. The study coordinator has the final responsibility.

Ethics approval has been obtained from the local Ethics Committee prior to the start of the study (see $\{24\}$ ). Technical know-how is mainly outsourced to the service units of the messenger service grape.

\section{Composition of the data monitoring committee, its role and reporting structure $\{21 \mathrm{a}\}$}

SPIRIT guidance: Composition of data monitoring committee (DMC); summary of its role and reporting structure; statement of whether it is independent from the sponsor and competing interests; and reference to where further details about its charter can be found, if not in the protocol. Alternatively, an explanation of why a DMC is not needed.

A Data Monitoring Committee (DMC) has been established (EH, AEZ, UH, JG).

On the one hand, the role of the committee members (AEZ, JG) is to conduct weekly checks of the communication protocols and to identify potential errors in measurement or possible anomalies in the behaviors of participants and moderators.

In addition, members of the $\mathrm{DMC}(\mathrm{EH}, \mathrm{UH})$ routinely check that adequate procedures are in place to ensure that the data remain confidential and that there are no data breaches. If security problems are identified, the Data Monitoring Committee informs the other study members or, depending on the severity of the problem, also the funding agency, and takes action to fix the problem.

\section{Adverse event reporting and harms $\{22\}$}

SPIRIT guidance: Plans for collecting, assessing, reporting, and managing solicited and spontaneously reported adverse events and other unintended effects of trial interventions or trial conduct.

Although we consider specific risks for participation very low, a system for collecting, assessing, reporting, and managing adverse events will be implemented. Every potential adverse event will be documented in detail. The study coordinator will immediately inform the Ethics Committee of Lower Austria and the study's insurance company if necessary.

If a problematic situation arises in an IMS group such as bullying, depression, racial/sexist/homophobic etc. statements, extensive sharing of fake news, commercials and the like, the moderator can try to solve it using the communication strategies learned during the training, discuss it with the dietitian and/or other moderators, or hand over the problem to the dietitian and/or the study staff. Furthermore, the study team will control the communication data for problematic situations at regular intervals. The dietitians will address this topic during the monthly meetings with the moderators as part of the process evaluation.

\section{Frequency and plans for auditing trial conduct $\{23\}$}

SPIRIT guidance: Frequency and procedures for auditing trial conduct, if any, and whether the process will be independent from investigators and the sponsor.

Not applicable as there are no planned audits.

Plans for communicating important protocol amendments to relevant parties (e.g. trial participants, ethical committees) \{25\} 
SPIRIT guidance: Plans for communicating important protocol modifications (eg, changes to eligibility criteria, outcomes, analyses) to relevant parties (eg, investigators, REC/IRBs, trial participants, trial registries, journals, regulators).

Approval for protocol modification will be sought for from the Ethics Committee of Lower Austria as well as the funding organization (Gesellschaft für Forschungsförderung Niederösterreich m.b.H, Austria). Upon approval of all changes, they will be noted in the study registration. If protocol modifications differ from what was explained to participants when signing the informed consent, the participants will be informed immediately about all changes and re-consent will be sought by a study staff member (dietitian or nutritionist).

\section{Dissemination plans $\{31 \mathrm{a}\}$}

SPIRIT guidance: Plans for investigators and sponsor to communicate trial results to participants, healthcare professionals, the public, and other relevant groups (eg, via publication, reporting in results databases, or other data sharing arrangements), including any publication restrictions.

The consortium has developed a dissemination plan which defines all relevant stakeholders and appropriate dissemination strategies as well as authorship guidelines (77) visualized in a GANTT chart including when to conduct which dissemination activity, and another tool for the documentation of all activities. The dissemination plan includes scientific as well as general dissemination activities.

All dissemination activities will be documented and reported by the responsible consortium member. When it comes to scientific dissemination, the study protocol and the study results will be published in peer-reviewed open access journals. The study and its results will be presented at scientific conferences. Various activities are planned to disseminate generally, e.g., at special events like the "European Researchers' Night" or at the "World Diabetes Day", study website posts, press releases or radio and TV contributions.

\section{Discussion}

Trials guidance: This should include a discussion of any practical or operational issues involved in performing the study and any issues not covered in other sections.

Diabetes mellitus type 2 is one of the major global causes of disability and mortality. During the past few decades, the prevalence of diabetes increased dramatically all over the world and rising numbers are expected for the next decades $(1,2)$.

Additionally, data indicate that the COVID-19 pandemic will worsen the global diabetes burden because of the bi-directional relationship between these two diseases: on the one hand, diabetes is associated with a poor prognosis of COVID-19, and COVID-19 patients with diabetes are more likely to face uncontrolled hyperglycemia or acute hyperglycemic crisis. On the other hand, a recent meta-analysis with approximately 3,700 participants shows a pooled proportion of $14.4 \%$ for newly diagnosed diabetes in hospitalized COVID-19 patients (78). Furthermore, the COVID-19 pandemic is suspected to have long-term effects on the prevalence of diabetes mellitus type 2 because of the lockdowns in almost every country. This unique situation has led to harmful effects such as increased calorie intake, reduced physical activity, increased screen time, weight gain, depression, boredom, disrupted sleep pattern, anxiety, and stress $(79,80)$. The majority of these effects are well-known risk factors for the development of diabetes mellitus type 2 (10).

This situation highlights the importance of appropriate diabetes management. DSMES is a key component of successful diabetes management and it is highly relevant to maintain the positive results in the long run. Therefore, DiabPeerS aims to improve diabetes selfmanagement leading to better glycemic control by implementing a peer-supported IMS intervention in addition to standard care. IMS provides several benefits:

1. IMS can be used time- and location-independently, which leads to higher participation rates of people with diabetes mellitus type 2 in diabetes self-management support offers.

2. IMS has a low threshold when exchange with others is needed.

3. IMS support and exchange can be provided immediately.

4. the majority of patients with diabetes already use IMS, which means that no technology barrier is perceived.

5. IMS groups can be supervised by different health professionals at the same time or used to to share workload or offer additional support. The additional support is an option of information verification, which increases the quality of the peer support.

6. IMS can help to lower costs for participants as well as for the healthcare/social system. 
To ensure positive effects of peer-supported IMS interventions, participants, moderators and health professionals should undergo adequate training because IMS demands very specific communication, e.g., engaging and motivating wording, emojis or short messages. Therefore, the DiabPeerS study has developed specific trainings for participants, moderators, and dietitians. Information concerning these trainings will be published after the end of the study.

Based on the multifactorial origin of diabetes mellitus type 2 and the need for holistic therapeutic approaches, the DiabPeerS study consists of an interdisciplinary team and looks at the disease requirements as well as the peer-supported IMS intervention from different scientific angles by adopting a mixed-methods approach. On the one hand, the study team will gain new insights concerning effective strategies to support diabetes disease management and improve disease-related outcomes. On the other hand, the IMS strategy and the trial outcomes can be analyzed in detail, e.g., topics can be identified which lead to increased discussion and exchange of participants or in-depth analysis of the results of the quantitative data.

Besides the undoubted negative consequences of the COVID-19 pandemic, people all over the world are now used to communicating online, which has led to a digital boost in the healthcare sector. As a consequence, new digital therapeutic and/or supportive approaches to various diseases - especially non-communicable diseases - will play an even greater role in the future. DiabPeerS is intended to make a relevant contribution to future high-quality online services for patients.

\section{Trial status}

Trials guidance: Authors should report the protocol version number and date, the date recruitment began, and the approximate date when recruitment will be completed.

This is the protocol version 5 from 31.07.2021. Recruitment will start in August 2021 and will end in September 2022.

\section{Abbreviations}

ADA: Aktive Diabetiker Austria; ANCOVA: Analysis of Covariance; BFI-2: Big Five Inventory German; BIA: Bioelectrical Impedance Analysis; COVID-19: Coronavirus SARS-CoV-2; DEGS-FFQ: Studie zur Gesundheit Erwachsener in Deutschland Food Frequency Questionnaire; DiabPeerS: Improving glycemic control in patients with type 2 diabetes mellitus through a peer support instant messaging service intervention; DDS: Diabetes Distress Scale; DEGES-FFQ: German Health Examination Survey for Adults 2008-2011 Food Frequency Questionnaire; DKT: Diabetes Knowledge Test; DMC: Data Monitoring Committee; DSM-IV: Diagnostic and Statistical Manual of Mental Disorders; DSME: diabetes self-management education; DSMES: diabetes self-management education and support; EUR: Euro; FoKo database: Folgekosten database; F-SozU: Fragebogen zur Sozialen Unterstützung; GSE: General Self-Efficacy Scale; $\mathrm{HbA}_{1 \mathrm{c}}$ : glycated hemoglobin; HDL: high-density lipoprotein; IMS: Instant Messaging Service; IPAQ-SF: International Physical Activity Questionnaire Short Form; IT: information technology; LDL: low-density lipoprotein; MCAR: missing completely at random; MET: metabolic equivalent of task; mHealth: Mobile Health; ÖDV: Österreichische Diabetikervereinigung; PHQ-9: Patient Health Questionnaire-9; RCT: randomized controlled trials; SDSCA-G: Summary of Diabetes Self-Care Activities German; SF-12: Short-Form-Health Survey; TV: television; USA: United States of America; USD: US Dollar

\section{Declarations}

\section{Acknowledgements}

Trials guidance: Please acknowledge anyone who contributed towards the article who does not meet the criteria for authorship including anyone who provided professional writing services or materials. Authors should obtain permission to acknowledge from all those mentioned in the Acknowledgements section. See our editorial policies for a full explanation of acknowledgements and authorship criteria. If you do not have anyone to acknowledge, please write "Not applicable" in this section.

Group authorship (for manuscripts involving a collaboration group): if you would like the names of the individual members of a collaboration Group to be searchable through their individual PubMed records, please ensure that the title of the collaboration Group is included on the title page and in the submission system and also include collaborating author names as the last paragraph of the "Acknowledgements" section. Please add authors in the format First Name, Middle initial(s) (optional), Last Name. You can add institution or country information for each author if you wish, but this should be consistent across all authors. Please note that individual names may not be present in the PubMed record at the time a published article is initially included in PubMed as it takes PubMed additional time to code this information. 
The authors gratefully acknowledge the financial support of the Gesellschaft für Forschungsförderung Niederösterreich m.b.H (GFF) and the provincial government of Lower Austria through the (Life) Science Calls (Project ID LS18-021). We would like to thank the NÖ Landesgesundheitsagentur, the Austrian diabetes self-help groups and involved students for their support of this study. Furthermore, we acknowledge the permission to use the following questionnaires:

- Department of General Practice and Health Services Research, University Hospital Heidelberg, Heidelberg, Germany for the SDSCA-G:

- Kamradt M, Bozorgmehr K, Krisam J, Freund T, Kiel M, Qreini M, et al. Assessing self-management in patients with diabetes mellitus type 2 in Germany: validation of a German version of the Summary of Diabetes Self-Care Activities measure (SDSCA-G). Health Qual Life Outcomes. 2014 Dec 18;12:185.

- Department of Clinical Pharmacology and Pharmacoepidemiology, Heidelberg University, Germany for the A14-Scale:

- Jank S, Bertsche T, Schellberg D, Herzog W, Haefeli WE. The A14-scale: development and evaluation of a questionnaire for assessment of adherence and individual barriers. Pharm World Sci 2009;31:426-31.

- Robert Koch Institute, Berlin, Germany for the DEGS-FFQ:

- Haftenberger M, Heuer T, Heidemann C, Kube F, Krems C, Mensink GB. Relative validation of a food frequency questionnaire for national health and nutrition monitoring. Nutr J. 2010 Dec;9(1):36.

- Michigan Diabetes Research Center, Michigan, USA for the Diabetes Knowledge Scale. The project was supported by Grant Number P30DK020572 (MDRC) from the National Institute of Diabetes and Digestive and Kidney Diseases

- Collins GS, Mughal S, Barnett AH, Fitzgerald J, Lloyd CE. Modification and validation of the Revised Diabetes Knowledge Scale. Diabet Med. 2010.

Finally, we thank for the opportunity to use the following questionnaires

- Schwarzer R, Jerusalem M. Generalized Self-Efficacy scale. In: Measures in health psychology: A user's portfolio Causal and control beliefs. Winsor: NFER-NELSON; 1995. p. 35-7.

- Kroenke K, Spitzer RL, Williams JB. The PHQ-9: validity of a brief depression severity measure. J Gen Intern Med. 2001 Sep;16(9):606-13.

- Polonsky WH, Fisher L, Earles J, Dudl RJ, Lees J, Mullan J, et al. Assessing Psychosocial Distress in Diabetes: Development of the Diabetes Distress Scale. Diabetes Care. 2005 Mar 1;28(3):626-31

- IPAQ International Questionnaire. Downloadable questionnaires [Internet]. [cited 2018 Aug 31]. Available from: https://sites.google.com/site/theipaq/questionnaire_links

- Danner D, Rammstedt B, Bluemke M, Treiber L, Berres S, Soto C, et al. Die deutsche Version des Big Five Inventory 2 (BFI-2). Zusammenstellung Sozialwissenschaftlicher Items Skalen ZIS [Internet]. 2016 [cited 2021 May 11]; Available from: http://zis.gesis.org/Doild/zis247

\section{Authors' contributions $\{31 \mathrm{~b}\}$}

SPIRIT guidance: [31b] - Authorship eligibility guidelines and any intended use of professional writers.

Trials guidance: The individual contributions of authors to the manuscript should be specified in this section. Guidance and criteria for authorship can be found in our editorial policies. Please use initials to refer to each author's contribution in this section, for example: "AB is the Chief Investigator; she conceived the study, led the proposal and protocol development. CD contributed to study design and to development of the proposal. EF was the lead trial methodologist. All authors read and approved the final manuscript."

EH is the study coordinator. EH, JG, MW, DWK and JP conceived the study and led the proposal and protocol development. EH and JG wrote the first draft of the manuscript. JP edited and made substantial contributions to the manuscript. EH is responsible for study management, measurements and data management. UH, AEZ, EH, JG, DWK, WK, SS developed the IMS strategy and the trainings. JG and AEZ will be responsible for interpreting communication data. UH is the dietitian in contact with moderators. MW and WK are the clinicians responsible for analyzing biochemical parameters. PK and EG will carry out the FoKo database query and contact potential participants 
as well as internists and physicians in Lower Austria. SS will be responsible for statistical analysis. All authors read and approved the final manuscript.

\section{Funding $\{4\}$}

SPIRIT guidance: Sources and types of financial, material, and other support.

Trials guidance: All sources of funding for the research reported should be declared. You will be required to include a copy of the original funding document and an English translation of this document as an additional file on submission, which will be checked against this declaration. The role of the funding body in the design of the study and collection, analysis, and interpretation of data and in writing the manuscript should be declared.

This study is publicly funded by the Gesellschaft für Forschungsförderung Niederösterreich m.b.H, Austria (Life Science Call 2018, LS18021). The funding organization has no influence on the design, collection, analysis, and interpretation of data, or the writing of the manuscript.

\section{Availability of data and materials $\{29\}$}

SPIRIT guidance: Statement of who will have access to the final trial dataset, and disclosure of contractual agreements that limit such access for investigators.

Trials guidance: Please do not include any baseline or pilot data in your study protocol. The Editorial Office will ask you to remove this if it is included. Please declare here who will have access to the final trial dataset and disclose contractual agreements that limit such access for investigators.

The Data Management Coordinating Center (EH, UH, AEZ, JG) will oversee the intra-study data sharing process, with input from the Data Management Subcommittee.

Data sharing, especially with the funding agency, will be done in exclusively encrypted form. Also, only the encrypted data will be used for any publications.

Medical samples will be destroyed after the analyses according to the quality criteria of ISO 9001 and will not be stored for further examinations.

The collected data and backup copies are protected by passwords and are also only accessible to those persons who are entrusted with their processing and evaluation.

For evaluation and publication, the data will be used in anonymized form only.

(1) Personal data will be kept separately from collected measurement data.

(2) An ID code is created for each participant (encryption by means of sequential numbering, from which only the assignment to the corresponding group is possible, but no conclusion regarding the identity of the participant).

\section{Ethics approval and consent to participate $\{24\}$}

SPIRIT guidance: Plans for seeking research ethics committee/institutional review board (REC/IRB) approval.

Trials guidance: Trials do not consider study protocols for studies without ethical approval. You will be required to provide a copy of the original ethical approval document and an English translation of this document as an additional file on submission, which will be checked against this declaration. The name of the ethics committee that approved the study and the committee's reference number (if applicable) should be declared. Details of authors' intentions to obtain consent to participate in the study from participants (or their parent or legal guardian in the case of children under 16) should be declared. "eg. ABC Ethical Review Board ABC123456. Written, informed consent to participate will be obtained from all participants"

This study was approved by the Ethics Committee of Lower Austria (Ethics number:

GS4-EK-4/569-2018). Written, informed consent to participate will be obtained from all participants.

\section{Consent for publication $\{32\}$}


SPIRIT guidance: Model consent form and other related documentation given to participants and authorised surrogates.

Trials guidance: Please do not include any baseline or pilot data in your study protocol. The Editorial Office will ask you to remove this if it is included. If you have included any details, images or videos relating to an individual person, written informed consent for the publication of these details must be obtained from that person (or their parent or legal guardian in the case of children under 18) and declared in this section. Please also state whether you will be willing to provide a model consent form on request. If this section does not apply, please state "Not applicable".

Not applicable.

\section{Competing interests $\{28\}$}

SPIRIT guidance: Financial and other competing interests for principal investigators for the overall trial and each study site.

Trials guidance: All financial and non-financial competing interests must be declared in this section. See our editorial policies for a full explanation of competing interests. If you are unsure whether you or any of your co-authors have a competing interest please contact the editorial office. Please use the authors initials to refer to each authors' competing interests in this section. If you do not have any competing interests, please state: "The authors declare that they have no competing interests" in this section.

The authors declare that they have no competing interests.

\section{References}

1. World Health Organization, editor. Global report on diabetes. Geneva: WHO Press, World Health Organization; 2016.86 p.

2. International Diabetes Federation. IDF Diabetes Atlas. Ninth edition 2019 [Internet]. 2019 [cited 2020 Nov 20]. Available from: https://www.diabetesatlas.org/upload/resources/material/20200302_133351_IDFATLAS9e-final-web.pdf

3. Schmutterer I, Delcour J, Griebler R. Österreichischer Diabetesbericht 2017. Wien: Bundesministerium für Gesundheit und Frauen; 2017.

4. Österreichische Diabetes Gesellschaft M. Diabetes mellitus - Anleitungen für-die Praxis. Überarbeitete und erweiterte Fassung 2019. 2019;131(Suppl 1):1-246.

5. Bommer C, Heesemann E, Sagalova V, Manne-Goehler J, Atun R, Bärnighausen T, et al. The global economic burden of diabetes in adults aged 20-79 years: a cost-of-illness study. Lancet Diabetes Endocrinol. 2017;5(6):423-30.

6. American Diabetes Association. Economic Costs of Diabetes in the U.S. in 2017. Diabetes Care. 2018;41(5):917-28.

7. Funnell MM, Anderson RM. The Problem With Compliance in Diabetes. JAMA. 2000 ;284(13):1709.

8. American Diabetes Association. 5. Facilitating Behavior Change and Well-being to Improve Health Outcomes: Standards of Medical Care in Diabetes-2020. Diabetes Care. 2020;43(Supplement 1):S48-65.

9. International Diabetes Federation. Global Guideline for Type 2 Diabetes [Internet]. 2012. Available from: https://www.idf.org/component/attachments/attachments.html?id=725\&task=download

10. Paulweber B, Valensi P, Lindström J, Lalic NM, Greaves CJ, McKee M, et al. A European Evidence-Based Guideline for the Prevention of Type 2 Diabetes. Horm Metab Res. 2010;42(S 1):S3-36.

11. Powers MA, Bardsley JK, Cypress M, Funnell MM, Harms D, Hess-Fischl A, et al. Diabetes Self-management Education and Support in Adults With Type 2 Diabetes: A Consensus Report of the American Diabetes Association, the Association of Diabetes Care \&amp; Education Specialists, the Academy of Nutrition and Dietetics, the American Academy of Family Physicians, the American Academy of PAs, the American Association of Nurse Practitioners, and the American Pharmacists Association. Diabetes Care. 2020;43(7):1636-49.

12. Mulcahy K, Maryniuk M, Peeples M, Peyrot M, Tomky D, Weaver T, et al. Diabetes self-management education core outcomes measures. Diabetes Educ. 2003;29(5):768-70, 773-84, 787-788 passim.

13. Bekele BB, Negash S, Bogale B, Tesfaye M, Getachew D, Weldekidan F, et al. Effect of diabetes self-management education (DSME) on glycated hemoglobin ( $\mathrm{HbA} 1 \mathrm{c})$ level among patients with T2DM: Systematic review and meta-analysis of randomized controlled trials. Diabetes Metab Syndr. 2021;15(1):177-85.

14. Odgers-Jewell K, Ball LE, Kelly JT, Isenring EA, Reidlinger DP, Thomas R. Effectiveness of group-based self-management education for individuals with Type 2 diabetes: a systematic review with meta-analyses and meta-regression. Diabet Med J Br Diabet Assoc.

2017;34(8):1027-39.

Page 21/26 
15. Tanaka R, Shibayama T, Sugimoto K, Hidaka K. Diabetes self-management education and support for adults with newly diagnosed type 2 diabetes mellitus: A systematic review and meta-analysis of randomized controlled trials. Diabetes Res Clin Pract. 2020;169:108480.

16. World Health Organization. Use of glycated haemoglobin (HbA1c) in the diagnosis of diabetes mellitus. Diabetes Res Clin Pract. 2011;93(3):299-309.

17. He X, Li J, Wang B, Yao Q, Li L, Song R, et al. Diabetes self-management education reduces risk of all-cause mortality in type 2 diabetes patients: a systematic review and meta-analysis. Endocrine. 2017;55(3):712-31.

18. Minet L, Møller S, Vach W, Wagner L, Henriksen JE. Mediating the effect of self-care management intervention in type 2 diabetes: a meta-analysis of 47 randomised controlled trials. Patient Educ Couns. 2010;80(1):29-41.

19. Steinsbekk A, Rygg LØ, Lisulo M, Rise MB, Fretheim A. Group based diabetes self-management education compared to routine treatment for people with type 2 diabetes mellitus. A systematic review with meta-analysis. BMC Health Serv Res. 2012;12:213.

20. Norris SL, Lau J, Smith SJ, Schmid CH, Engelgau MM. Self-management education for adults with type 2 diabetes: a meta-analysis of the effect on glycemic control. Diabetes Care. 2002;25(7):1159-71.

21. Shumaker SA, Brownell A. Toward a Theory of Social Support: Closing Conceptual Gaps. J Soc Issues. 1984;40(4):11-36.

22. Song Y, Nam S, Park S, Shin I-S, Ku BJ. The Impact of Social Support on Self-care of Patients With Diabetes: What Is the Effect of Diabetes Type? Systematic Review and Meta-analysis. Diabetes Educ. 2017;43(4):396-412.

23. Wang X, He L, Zhu K, Zhang S, Xin L, Xu W, et al. An integrated model to evaluate the impact of social support on improving selfmanagement of type 2 diabetes mellitus. BMC Med Inform Decis Mak [Internet]. 2019 Oct 22 [cited 2021 Apr 9];19. Available from: https://www.ncbi.nlm.nih.gov/pmc/articles/PMC6805520/

24. Powers MA, Bardsley J, Cypress M, Duker P, Funnell MM, Fischl AH, et al. Diabetes Self-management Education and Support in Type 2 Diabetes: A Joint Position Statement of the American Diabetes Association, the American Association of Diabetes Educators, and the Academy of Nutrition and Dietetics. Clin Diabetes Publ Am Diabetes Assoc. 2016;34(2):70-80.

25. Dennis C-L. Peer support within a health care context: a concept analysis. Int J Nurs Stud. 2003;40(3):321-32.

26. Gatlin TK, Serafica R, Johnson M. Systematic review of peer education intervention programmes among individuals with type 2 diabetes. J Clin Nurs. 2017;26(23-24):4212-22.

27. Patil SJ, Ruppar T, Koopman RJ, Lindbloom EJ, Elliott SG, Mehr DR, et al. Peer Support Interventions for Adults With Diabetes: A MetaAnalysis of Hemoglobin A1c Outcomes. Ann Fam Med. 2016;14(6):540-51.

28. Patil SJ, Ruppar T, Koopman RJ, Lindbloom EJ, Elliott SG, Mehr DR, et al. Effect of peer support interventions on cardiovascular disease risk factors in adults with diabetes: a systematic review and meta-analysis. BMC Public Health. 2018;18(1):398.

29. Dale JR, Williams SM, Bowyer V. What is the effect of peer support on diabetes outcomes in adults? A systematic review. Diabet Med J Br Diabet Assoc. 2012;29(11):1361-77.

30. Johansson T, Keller S, Winkler H, Ostermann T, Weitgasser R, Sönnichsen AC. Effectiveness of a Peer Support Programme versus Usual Care in Disease Management of Diabetes Mellitus Type 2 regarding Improvement of Metabolic Control: A Cluster-Randomised Controlled Trial. J Diabetes Res [Internet]. 2016 [cited 2021 Apr 9];2016. Available from: https://www.ncbi.nlm.nih.gov/pmc/articles/PMC4698561/

31. World Health Organization. mHealth: new horizons for health through mobile technologies: second global survey on eHealth [Internet]. World Health Organization; 2011 [cited 2021 Mar 19]. Available from: https://apps.who.int/iris/handle/10665/44607

32. Eberle C, Stichling S, Löhnert M. Diabetology 4.0: Scoping Review of Novel Insights and Possibilities Offered by Digitalization. J Med Internet Res. 2021;23(3):e23475.

33. Kitsiou S, Paré G, Jaana M, Gerber B. Effectiveness of mHealth interventions for patients with diabetes: An overview of systematic reviews. PloS One. 2017;12(3):e0173160.

34. Wang Y, Min J, Khuri J, Xue H, Xie B, A Kaminsky L, et al. Effectiveness of Mobile Health Interventions on Diabetes and Obesity Treatment and Management: Systematic Review of Systematic Reviews. JMIR MHealth UHealth [Internet]. 2020 [cited 2021 Apr 9];8(4). Available from: https://www.ncbi.nlm.nih.gov/pmc/articles/PMC7218595/

35. Morris C, Scott RE, Mars M. Security and Other Ethical Concerns of Instant Messaging in Healthcare. Stud Health Technol Inform. 2018;254:77-85.

36. Gabarron E, Årsand E, Wynn R. Social Media Use in Interventions for Diabetes: Rapid Evidence-Based Review. J Med Internet Res. 2018;20(8):e10303. 
37. Vorderstrasse A, Lewinski A, Melkus GD, Johnson C. Social Support for Diabetes Self-Management via eHealth Interventions. Curr Diab Rep. 2016;16(7):56.

38. Statistik Austria. IKT-Einsatz in Haushalten [Internet]. Erhebung über den IKT-Einsatz in Haushalten 2020.2020 [cited 2021 Apr 9]. Available from: http://www.statistik.at/web_de/statistiken/energie_umwelt_innovation_mobilitaet/informationsgesellschaft/ikteinsatz_in_haushalten/024571.html

39. Tang TS, Funnell M, Sinco B, Piatt G, Palmisano G, Spencer MS, et al. Comparative effectiveness of peer leaders and community health workers in diabetes self-management support: results of a randomized controlled trial. Diabetes Care. 2014;37(6):1525-34.

40. Giordano V, Koch H, Godoy-Santos A, Dias Belangero W, Esteves Santos Pires R, Labronici P. WhatsApp Messenger as an Adjunctive Tool for Telemedicine: An Overview. Interact J Med Res. 2017;6(2):e11.

41. Kleinberger JW, Pollin TI. Personalized medicine in diabetes mellitus: current opportunities and future prospects. Ann N Y Acad Sci. 2015;1346(1):45-56.

42. Scheen AJ. Precision medicine: The future in diabetes care? Diabetes Res Clin Pract. 2016;117:12-21.

43. Dugas M, Crowley K, Gao GG, Xu T, Agarwal R, Kruglanski AW, et al. Individual differences in regulatory mode moderate the effectiveness of a pilot mHealth trial for diabetes management among older veterans. PLOS ONE. 2018;13(3):e0192807.

44. Klein WMP, Rothman AJ, Cameron LD. Theoretical innovations in social and personality psychology and implications for health: introduction to special issue. Health Psychol Off J Div Health Psychol Am Psychol Assoc. 2013;32(5):457-9.

45. Montag C, Błaszkiewicz K, Sariyska R, Lachmann B, Andone I, Trendafilov B, et al. Smartphone usage in the 21st century: who is active on WhatsApp? BMC Res Notes. 2015;8(1):331.

46. Amt der NÖ Landesregierung. Bevölkerungsstruktur 2020 [Internet]. Zahlen Fakten. 2021 [cited 2021 May 28]. Available from: https://www.noe.gv.at/noe/Zahlen-Fakten/Bevstruktur_Alter_2020.xlsx

47. Statistik Austria. Bevölkerung [Internet]. 2021 [cited 2021 Jun 10]. Available from: http://pic.statistik.at/web_de/statistiken/menschen_und_gesellschaft/bevoelkerung/index.html

48. Smith PC, Schmidt SM, Allensworth-Davies D, Saitz R. A Single-Question Screening Test for Drug Use in Primary Care. Arch Intern Med. 2010;170(13):1155-60.

49. American Diabetes Association. 4. Lifestyle Management: Standards of Medical Care in Diabetes-2018. Diabetes Care. 2018;41(Supplement 1):S38-50.

50. Atreja A, Bellam N, Levy SR. Strategies to Enhance Patient Adherence: Making it Simple. Medscape Gen Med. 2005;7(1):4.

51. Matsui D. Strategies to Measure and Improve Patient Adherence in Clinical Trials. Pharm Med. 2009;23(5):289-97.

52. Riedl R, Robausch M, Berghold A. The Evaluation of the Effectiveness of Austrians Disease Management Program in Patients with Type 2 Diabetes Mellitus - A Population-Based Retrospective Cohort Study. Reboldi G, editor. PLOS ONE. 2016;11(8):e0161429.

53. Fydrich T, Sommer G, Brähler E. Fragebogen zu Sozialen Unterstützung. Hogrefe; 2007.

54. Schwarzer R, Jerusalem M. Generalized Self-Efficacy scale. In: Measures in health psychology: A user's portfolio Causal and control beliefs. Winsor: NFER-NELSON; 1995. p. 35-7.

55. Kroenke K, Spitzer RL, Williams JB. The PHQ-9: validity of a brief depression severity measure. J Gen Intern Med. 2001;16(9):606-13.

56. Polonsky WH, Fisher L, Earles J, Dudl RJ, Lees J, Mullan J, et al. Assessing Psychosocial Distress in Diabetes: Development of the Diabetes Distress Scale. Diabetes Care. 2005;28(3):626-31.

57. Morfeld M, Kirchberger I, Bullinger M. SF-36 Fragebogen zum Gesundheitszustand. 2., ergänzte und überarbeitete Auflage. Hogrefe; 2011.

58. Collins GS, Mughal S, Barnett AH, Fitzgerald J, Lloyd CE. Modification and validation of the Revised Diabetes Knowledge Scale. Diabet Med. 2010.

59. Jank S, Bertsche T, Schellberg D, Herzog W, Haefeli WE. The A14-scale: development and evaluation of a questionnaire for assessment of adherence and individual barriers. Pharm World Sci PWS. 2009;31(4):426-31.

60. Haftenberger M, Heuer T, Heidemann C, Kube F, Krems C, Mensink GB. Relative validation of a food frequency questionnaire for national health and nutrition monitoring. Nutr J. 2010;9(1):36.

61. Robert Koch Institut. DEGS-Ernährungsfragebogen [Internet]. 2011. Available from: https://www.rki.de/DE/Content/Gesundheitsmonitoring/Studien/Methodik/Befragungsmethoden/ernaehrung/DEGS_FFQ_inhalt.html

62. Latza U, Hoffmann W, Terschüren C, Chang-Claude J, Kreuzer M, Schaffrath Rosario A, et al. Erhebung, Quantifizierung und Analyse der Rauchexposition in epidemiologischen Studien [Internet]. Berlin: Robert Koch-Institut; 2005 [cited 2021 May 27]. Available from: 
https://www.rki.de/DE/Content/Gesundheitsmonitoring/Themen/Rauchen/Rauchexposition_epidstudien.pdf?_blob=publicationFile

63. IPAQ International Questionnaire. Downloadable questionnaires [Internet]. [cited 2018 Aug 31]. Available from: https://sites.google.com/site/theipaq/questionnaire_links

64. Lee PH, Macfarlane DJ, Lam TH, Stewart SM. Validity of the International Physical Activity Questionnaire Short Form (IPAQ-SF): a systematic review. Int J Behav Nutr Phys Act. 2011;8:115.

65. Kamradt M, Bozorgmehr K, Krisam J, Freund T, Kiel M, Qreini M, et al. Assessing self-management in patients with diabetes mellitus type 2 in Germany: validation of a German version of the Summary of Diabetes Self-Care Activities measure (SDSCA-G). Health Qual Life Outcomes. 2014;12:185.

66. United Nations Economic Commission for Europe. Conference of European Statisticians Recommendations for the 2020 Censuses of Population and Housing [Internet]. New York and Geneva; 2015 [cited 2021 May 11]. Available from: https://unece.org/fileadmin/DAM/stats/publications/2015/ECECES41_EN.pdf

67. Danner D, Rammstedt B, Bluemke M, Treiber L, Berres S, Soto C, et al. Die deutsche Version des Big Five Inventory 2 (BFI-2). Zusammenstellung Sozialwissenschaftlicher Items Skalen ZIS [Internet]. 2016 [cited 2021 May 11]; Available from: http://zis.gesis.org/Doild/zis247

68. Hughes DJ, Rowe M, Batey M, Lee A. A tale of two sites: Twitter vs. Facebook and the personality predictors of social media usage. Comput Hum Behav. 2012;28(2):561-9.

69. Pittman M. Phoneliness: Examining the relationships between mobile social media, personality and loneliness. 2017 Sep 6 [cited 2021 Jun 15]; Available from: https://scholarsbank.uoregon.edu/xmlui/handle/1794/22699

70. Röhner J, Schütz A. Psychologie der Kommunikation (Basiswissen Psychologie). 2nd Edition. Springer VS; 2016.48 p.

71. Faul F, Erdfelder E, Lang A-G, Buchner A. G*Power 3: a flexible statistical power analysis program for the social, behavioral, and biomedical sciences. Behav Res Methods. 2007;39(2):175-91.

72. Azami G, Soh KL, Sazlina SG, Salmiah MS, Aazami S, Mozafari M, et al. Effect of a Nurse-Led Diabetes Self-Management Education Program on Glycosylated Hemoglobin among Adults with Type 2 Diabetes. J Diabetes Res. 2018;2018:4930157.

73. Gagliardino JJ, Arrechea V, Assad D, Gagliardino GG, González L, Lucero S, et al. Type 2 diabetes patients educated by other patients perform at least as well as patients trained by professionals: Peer Diabetes Education. Diabetes Metab Res Rev. 2013;29(2):152-60.

74. Tshiananga JKT, Kocher S, Weber C, Erny-Albrecht K, Berndt K, Neeser K. The effect of nurse-led diabetes self-management education on glycosylated hemoglobin and cardiovascular risk factors: a meta-analysis. Diabetes Educ. 2012;38(1):108-23.

75. Bundesministerium Digitalisierung und Wirtschaftsstandort. Mother-Child-Booklet [Internet]. Available from: https://www.help.gv.at/Portal.Node/hlpd/public/content/143/Seite.1430100.html\#child

76. Field A. Discovering statistics using SPSS. Third edition. Sage; 2009.

77. Medical University of Vienna. Good Scientific Practice. Ethics in Science and Research. Guidelines of the Medical University of Vienna [Internet]. 2017 [cited 2021 May 10]. Available from:

https://www.meduniwien.ac.at/web/fileadmin/content/forschung/pdf/MedUni_Wien_GSP-Richtlinien_2017.pdf

78. Sathish T, Kapoor N, Cao Y, Tapp RJ, Zimmet P. Proportion of newly diagnosed diabetes in COVID-19 patients: A systematic review and meta-analysis. Diabetes Obes Metab. 2021;23(3):870-4.

79. Parekh N, Deierlein AL. Health behaviours during the coronavirus disease 2019 pandemic: implications for obesity. Public Health Nutr. 2020;1-5.

80. Zupo R, Castellana F, Sardone R, Sila A, Giagulli VA, Triggiani V, et al. Preliminary Trajectories in Dietary Behaviors during the COVID19 Pandemic: A Public Health Call to Action to Face Obesity. Int J Environ Res Public Health [Internet]. 2020 Oct [cited 2020 Nov 13];17(19). Available from: https://www.ncbi.nlm.nih.gov/pmc/articles/PMC7579065/

\section{Figures}




\begin{tabular}{|c|c|c|}
\hline & Assessed for eligibility $(n=)$ & \\
\hline & $\nabla$ & $\begin{array}{l}\text { Not eligible }(\mathrm{n}=) \\
\text { - Inclusion criteria not fulfilled } \\
\text { - Exclusion criteria met } \\
\text { - Participation refused }\end{array}$ \\
\hline & $\begin{array}{l}\text { Randomization } \\
\text { - Participants }(n=196) \\
\text { - Moderators }(n=9) \\
\end{array}$ & \\
\hline \multirow{2}{*}{\multicolumn{2}{|c|}{$\begin{array}{l}\text { Intervention group } \\
\text { - Participants }(n=98)+\text { moderators }(n=9) \\
\text { Subdivided into } 7 \text { peer-supported IMS groups } \\
\text { - } 14 \text { participants per group } \\
\text { - } 1 \text { moderator per group }\end{array}$}} & \multirow{2}{*}{$\begin{array}{l}\text { Control group } \\
\text { - Participants }(n=98)\end{array}$} \\
\hline & & \\
\hline \multicolumn{2}{|l|}{ Standard therapy } & Standard therapy \\
\hline
\end{tabular}

Figure 1

Flow diagram DiabPeerS 


\begin{tabular}{|c|c|c|c|c|c|c|}
\hline \multirow[b]{3}{*}{ TIMEPOINT } & \multicolumn{6}{|c|}{ STUDY PERIOD } \\
\hline & \multirow{2}{*}{$\begin{array}{c}\text { Enrollment } \\
-t\end{array}$} & \multirow{2}{*}{$\begin{array}{c}\text { Allocation } \\
0\end{array}$} & \multicolumn{4}{|c|}{ Post-allocation } \\
\hline & & & $\begin{array}{c}\text { Baseline } \\
\text { to }\end{array}$ & \begin{tabular}{|l|}
3 months \\
after the \\
start of \\
the inter- \\
vention $t$
\end{tabular} & \begin{tabular}{|c|} 
At the \\
end of \\
the 7 \\
months \\
of inter. \\
vention $t_{2}$ \\
\end{tabular} & $\begin{array}{l}\text { Follow- } \\
\text { up to }\end{array}$ \\
\hline \multicolumn{7}{|l|}{ ENROLLMENT: } \\
\hline Eligibility screen & $x$ & & & & & \\
\hline Informed consent & $x$ & & & & & \\
\hline Allocation & & $x$ & & & & \\
\hline \multicolumn{7}{|l|}{ INTERVENTIONS: } \\
\hline \\
\hline & & & & $\longrightarrow$ & \\
\hline \multicolumn{7}{|l|}{ 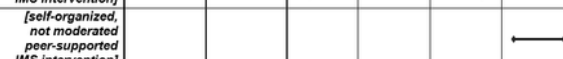 } \\
\hline \multicolumn{7}{|l|}{ ASSESSMENTS: } \\
\hline $\begin{array}{r}\text { [socio- } \\
\text { demographic } \\
\text { variables] }\end{array}$ & $x$ & & & & & \\
\hline $\begin{array}{r}\text { [Clinical } \\
\text { information] }\end{array}$ & $x$ & & & & & \\
\hline [personal traits\} & & & $\mathrm{x}$ & & & \\
\hline [Pregnancy control & & & $\mathrm{x}$ & $\mathrm{x}$ & $x$ & $\mathrm{x}$ \\
\hline $\begin{array}{l}\text { [social support: Fi } \\
\text { Sozul] }\end{array}$ & & & $\mathrm{x}$ & $x$ & $\mathrm{x}$ & $x$ \\
\hline [solf-efficacy: GSE] & & & $x$ & $x$ & $x$ & $x$ \\
\hline [depression: PHQ- & & & $x$ & $x$ & $x$ & $x$ \\
\hline [quality of life: SF. & & & $x$ & $x$ & $x$ & $x$ \\
\hline $\begin{array}{r}\text { [diabotes } \\
\text { knowledge: } D K T]\end{array}$ & & & $x$ & $x$ & $x$ & $x$ \\
\hline \multirow{2}{*}{$\begin{array}{r}\text { Imedication } \\
\text { adherence: A-14l| } \\
\text { Idiotary behavior, } \\
\text { alcohol } \\
\text { consumption: } \\
\text { DEGS-FFQ] }\end{array}$} & & & $x$ & $x$ & $x$ & $x$ \\
\hline & & & $x$ & $x$ & $x$ & $x$ \\
\hline $\begin{array}{l}\text { Ismoking: } \\
\text { Aktivrauchen- } \\
\text { Kurversion } \\
\text { (Ewrachsene) I }\end{array}$ & & & $\mathrm{x}$ & $\mathrm{x}$ & $x$ & $x$ \\
\hline $\begin{array}{l}\text { [physical activity: } \\
\text { [PA-SF] }\end{array}$ & & & $\mathrm{x}$ & $x$ & $\mathrm{x}$ & $x$ \\
\hline $\begin{array}{l}\text { ldiabotess self- } \\
\text { management } \\
\text { behaviors: SDSCA. }\end{array}$ & & & $\mathrm{x}$ & $x$ & $x$ & $x$ \\
\hline $\begin{array}{r}\text { [Clinical and } \\
\text { communication } \\
\text { visits over the past } \\
6 \text { months] }\end{array}$ & & & $x$ & $x$ & $x$ & $x$ \\
\hline [HDA1C] & & & $x$ & $x$ & $x$ & $x$ \\
\hline $\begin{array}{r}\text { [fasting blood } \\
\text { glucosel }\end{array}$ & & & $\mathrm{x}$ & $x$ & $\mathrm{x}$ & $x$ \\
\hline \multirow{2}{*}{ (total cholesterol] } & & & $\mathrm{x}$ & $x$ & $x$ & $x$ \\
\hline & & & $x$ & $x$ & $x$ & $x$ \\
\hline [HOL] & & & $x$ & $x$ & $x$ & $x$ \\
\hline \multirow{2}{*}{ [blood pressure] } & & & $x$ & $x$ & $x$ & $x$ \\
\hline & & & $x$ & $x$ & $x$ & $x$ \\
\hline [body height] & & & $\mathrm{x}$ & $x$ & $x$ & $x$ \\
\hline \multirow{2}{*}{ 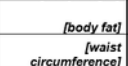 } & & & $x$ & $x$ & $x$ & $x$ \\
\hline & & & $\mathrm{x}$ & $x$ & $x$ & $x$ \\
\hline [communication & & & $x$ & $x$ & $x$ & $x$ \\
\hline
\end{tabular}

Figure 2

SPIRIT figure DiabPeerS 